\title{
WELL-POSEDNESS FOR GENERALIZED VARIATIONAL-HEMIVARIATIONAL INEQUALITIES WITH PERTURBATIONS IN REFLEXIVE BANACH SPACES
}

\author{
LU-CHUAN CENG, YUNG-YIH LUR AND CHING-FENG WEN
}

\begin{abstract}
In this paper, we consider an extension of well-posedness for a minimization problem to a class of generalized variational-hemivariational inequalities with perturbations in reflexive Banach spaces. We establish some metric characterizations for the $\alpha$ well-posed generalized variational-hemivariational inequality and give some conditions under which the generalized variational-hemivariational inequality is strongly $\alpha$-wellposed in the generalized sense. Under some mild conditions, we also prove the equivalence between the $\alpha$-well-posedness of the generalized variational-hemivariational inequality and the $\alpha$-well-posedness of the corresponding inclusion problem.
\end{abstract}

\section{Introduction}

In 1966, Tykhonov [24] first introduced a classical notion of well-posedness for a minimization problem, which has been known as the Tykhonov well-posedness. The minimization problem is said to be well-posed if there exists a unique minimizer and every minimizing sequence converges to the unique minimizer. Meantime, the concept of generalized Tykhonov well-posedness is introduced for the minimization problem, which means the existence of minimizers and the convergence of some subsequence of every minimizing sequence toward a minimizer. It is clear that the concept of well-posedness is inspired by numerical methods producing optimizing sequences for optimization problems and plays a crucial role in optimization theory. Because of its importance in optimization problems, various kinds of well-posedness for optimization problems have been introduced and studied by many mathematicians in the optimization research field. For more details, we refer to [11, 14, 29, 30] and the references therein.

Received March 21, 2017, accepted June 6, 2017. 2010 Mathematics Subject Classification. 49J40, 49K40, 90C31.

Key words and phrases. Generalized variational-hemivariational inequality, well-posedness, Clarke's generalized gradient, approximating sequence, inclusion problem.

This research was partially supported by the Innovation Program of Shanghai Municipal Education Commission (15ZZ068), Ph.D. Program Foundation of Ministry of Education of China (20123127110002) and Program for Outstanding Academic Leaders in Shanghai City (15XD1503100). Corresponding author: Ching-Feng Wen. 
Furthermore, it is well known that a differentiable minimization problem is closely related to a variational inequality of differential type. Naturally, the concept of well-posedness has been captured by many researchers to study variational inequalities. An initial notion of well-posedness for variational inequality is due to Lucchetti and Patrone [17]. They introduced the concept of well-posedness for a variational inequality and proved some related results by means of Ekeland's variational principle. Fang et al. [5, 6] generalized two kinds of well-posedness for a mixed variational inequality problem in Banach space, respectively. They established some metric characterizations of the two kinds of well-posedness for the mixed variational inequality, showed the equivalence of the two kinds of well-posedness among the mixed variational inequality problem, its corresponding inclusion problem and its corresponding fixed point problem, and derived some conditions under which the two kinds of well-posedness for the mixed variational inequality are equivalent to the existence and uniqueness of its solution. In recent years, the concept of well-posedness has been generalized to various kinds of well-posedness for different variational inequalities by many authors. Also, they established the metric characterizations for well-posed variational inequalities, the necessary and sufficient conditions of well-posedness for variational inequalities, and the links of well-posedness between variational inequalities and their related problems such as minimization problems, fixed pointed problems and inclusion problems. For further results on the well-posedness of variational inequalities, we refer to [10, 12, 13, 23, 33] and the references therein.

As an important and useful generalization of variational inequality, hemivariational inequality was first introduced in order to formulate variational principles involving nonconvex and nonsmooth energy functions, and investigated by Panagiotopoulos [21] using the mathematical concepts of the Clarke's generalized gradient for nonconvex and nondifferentiable functions [4]. The hemivariational inequalities have been proved very efficient to describe a variety of mechanical and engineering problems, e.g., non-monotone semipermeability problems, unilateral contact problems in nonlinear elasticity; see e.g., [1, 2, 9, 16, 18, 19, 20, 22]. It seems to be natural and easy to generalize the concept of well-posedness to hemivariational inequalities and most results on well-posedness for variational inequalities should hold for hemivariational inequalities under some similar conditions. However, it is not the truth. The Clarke's generalized directional derivative of a nonconvex and nonsmooth Lipschitz functional in hemivariational inequalities makes it much difficult. However, there are very few researchers extending the well-posedness to a hemivariational inequality.

In 1995, Goeleven and Mentagui [8] first introduced the well-posedness for a hemivariational inequality and presented some basic results concerning the well-posed hemivariational inequality. Recently, using the concept of approximating sequence, Xiao et al. [26, 
27] defined a concept of well-posedness for a hemivariational inequality and a variationalhemivariational inequality. They gave some metric characterizations for the well-posed hemivariational inequality and the well-posed variational-hemivariational inequality, and proved the equivalence of well-posedness between the hemivariational inequality and the corresponding inclusion problem. However, for the conditions of well-posedness for the hemivariational inequality and the variational-hemivariational inequality, Xiao et al. [26, 27] only gave a sufficient condition in Euclidean space $\mathbf{R}^{\mathbf{n}}$. In addition, for other recent works on the well-posedness for variational-hemivariational inequalities; see also e.g., [3, 34].

Let $X$ be a real reflexive Banach space with its dual $X^{*}$. We denote the duality pairing between $X$ and $X^{*}$ by $\langle\cdot, \cdot\rangle$, and the norm of Banach space $X$ by $\|\cdot\|$. In this paper, we always suppose that $F: X \rightarrow 2^{X^{*}}$ is a nonempty set-valued mapping, $T: X \rightarrow X^{*}$ is a perturbation, $G: X \rightarrow \mathbf{R} \cup\{+\infty\}$ is a proper, convex and lower semicontinuous functional, $J^{\circ}(\cdot, \cdot)$ stands for the Clarke's directional derivative of the locally Lipschitz functional $J: X \rightarrow \mathbf{R}$, and $f \in X^{*}$ is some given element. Denote by $\operatorname{dom} G$ the domain of functional $G$, i.e.,

$$
\operatorname{dom} G:=\{x \in X: G(x)<+\infty\} .
$$

Consider the following generalized variational-hemivariational inequality: find $x \in X$ such that for some $u \in F(x)$,

$$
\begin{aligned}
\text { GVHVI : } & \langle u+T x, y-x\rangle+J^{\circ}(x, y-x)+G(y)-G(x) \geq\langle f, y-x\rangle, \\
& \forall y \in X .
\end{aligned}
$$

In particular, if $F=A$ a single-valued mapping from $X$ to $X^{*}$, then the generalized variationalhemivariational inequality GVHVI reduces to the variational-hemivariational inequality VHVI considered in Xiao and Huang [26]. A concrete example of a variational-hemivariational inequality (see [20]) is the adhesive contact problem between a linear elastic body and a rubber support considered in [26], which is subject to a nonmonotone multivalued boundary condition.

In the present paper, we generalize the concept of well-posedness for the variationalhemivariational inequality with perturbation in Xiao and Huang [26] to the generalized variational-hemivariational inequality with perturbation, which includes, as special cases, the classical hemivariational inequality, the variational-hemivariational inequality, and the generalized mixed variational inequality. Under very mild conditions, we establish some metric characterizations for the $\alpha$-well-posed generalized variational-hemivariational inequality, and derive some conditions under which the generalized variational-hemivariational inequality is strongly $\alpha$-well-posed in the generalized sense. We also prove that the $\alpha$-wellposedness of the generalized variational-hemivariational inequality is equivalent to the $\alpha$ well-posedness of the corresponding inclusion problem. 


\section{Preliminaries}

Throughout this paper, unless stated otherwise, we always suppose that $X$ is a real reflexive Banach space and the norms of $X$ and its dual $X^{*}$ are denoted by the same symbol $\|\cdot\|$. Assume that $J: X \rightarrow \mathbf{R}$ is a locally Lipschitz functional on $X, x$ is a given point and $y$ is a vector in $X$. The Clarke's generalized directional derivative of $J$ at $x$ in the direction $y$, denoted by $J^{\circ}(x, y)$, is defined by

$$
J^{\circ}(x, y)=\limsup _{z \rightarrow x} \frac{J(z+\lambda y)-J(z)}{\lambda} .
$$

Let $G: X \rightarrow \mathbf{R} \cup\{+\infty\}$ be a proper, convex and lower semicontinuous functional. We denote by $\partial G(x): X \rightarrow 2^{X^{*}} \backslash\{\varnothing\}$ and $\bar{\partial} J(x): X \rightarrow 2^{X^{*}} \backslash\{\varnothing\}$ the subgradient of convex functional $G$ in the sense of convex analysis (see [36]) and the Clarke's generalized gradient of a locally Lipschitz functional $J$ (see [4]), respectively. That is,

$$
\partial G(x)=\left\{\varrho \in X^{*}: G(y)-G(x) \geq\langle\varrho, y-x\rangle, \forall y \in X\right\}
$$

and

$$
\bar{\partial} J(x)=\left\{\xi \in X^{*}: J^{\circ}(x, y) \geq\langle\xi, y\rangle, \forall y \in X\right\} .
$$

Remark 2.1 (see [1]). The Clarke's generalized gradient of a locally Lipschitz functional $J$ : $X \rightarrow \mathbf{R}$ at a point $x$ is given by

$$
\bar{\partial} J(x)=\partial\left(J^{\circ}(x, \cdot)\right)(0)
$$

Concerning the subgradient in the sense of convex analysis, the Clarke's generalized directional derivative and the Clarke's generalized gradient, we have the following basic properties (see e.g., $[1,4,18,20,36])$.

Proposition 2.1. Let $X$ be a Banach space and $G: X \rightarrow \mathbf{R} \cup\{+\infty\}$ be a convex and proper functional. Then we have the following properties of $\partial G$ :

(i) $\partial G(x)$ is convex and weak ${ }^{*}$-closed;

(ii) If $G$ is continuous at $x \in \operatorname{dom} G$, then $\partial G(x)$ is nonempty, convex, bounded, and weak* compact;

(iii) If $G$ is Gateaux differentiable at $x \in \operatorname{dom} G$, then $\partial G(x)=\{D G(x)\}$, where $D G(x)$ is the Gateaux derivative of $G$ at $x$.

Proposition 2.2. Let $X$ be a Banach space and $G_{1}, G_{2}: X \rightarrow \mathbf{R} \cup\{+\infty\}$ be two convex functionals. If there is a point $x_{0} \in \operatorname{dom} G_{1} \cap \operatorname{dom} G_{2}$ at which $G_{1}$ is continuous, then the following equation holds:

$$
\partial\left(G_{1}+G_{2}\right)(x)=\partial G_{1}(x)+\partial G_{2}(x), \quad \forall x \in X .
$$


Proposition 2.3. Let $X$ be a Banach space, $x, y \in X$ and $J$ be a locally Lipschitz functional defined on $X$. Then

(i) The function $y \mapsto J^{\circ}(x, y)$ is finite, positively homogeneous, subadditive and then convex on $X$;

(ii) $J^{\circ}(x, y)$ is upper semicontinuous as a function of $(x, y)$, as a function of $y$ alone, is Lipschitz continuous on $X$;

(iii) $J^{\circ}(x,-y)=(-J)^{\circ}(x, y)$;

(iv) $\bar{\partial} J(x)$ is a nonempty, convex, bounded and weak ${ }^{*}$-compact subset of $X^{*}$;

(v) For every $y \in X$, one has

$$
J^{\circ}(x, y)=\max \{\langle\xi, y\rangle: \xi \in \bar{\partial} J(x)\} ;
$$

(vi) The graph of the Clarke's generalized gradient $\bar{\partial} J(x)$ is closed in $X \times\left(w^{*}-X^{*}\right)$ topology, where $\left(w^{*}-X^{*}\right)$ denotes the space $X^{*}$ equipped with weak ${ }^{*}$ topology, i.e., if $\left\{x_{n}\right\} \subset X$ and $\left\{x_{n}^{*}\right\} \subset X^{*}$ are sequences such that $x_{n}^{*} \in \bar{\partial} J\left(x_{n}\right), x_{n} \rightarrow x$ in $X$ and $x_{n}^{*} \rightarrow x^{*}$ weakly in $X^{*}$, then $x^{*} \in \bar{\partial} J(x)$.

In the sequel, we recall some important definitions and useful results.

Definition 2.1. Let $X$ be a Banach space with its dual $X^{*}$ and $T$ a single-valued operator from $X$ to its dual space $X^{*}$. T is said to be monotone, if

$$
\langle T x-T y, x-y\rangle \geq 0, \quad \forall x, y \in X .
$$

Definition 2.2. Let $X$ be a Banach space with its dual $X^{*}$ and $F: X \rightarrow 2^{X^{*}}$ a nonempty multivalued operator from $X$ to $X^{*} . F$ is said to be monotone, if

$$
\langle u-v, x-y\rangle \geq 0, \quad \forall x, y \in X, u \in F(x), v \in F(y) .
$$

Let $A_{1}, A_{2}$ be nonempty subsets of a normed vector space $(X,\|\cdot\|)$. The Hausdorff metric $\mathscr{H}(\cdot, \cdot)$ between $A_{1}$ and $A_{2}$ is defined by

$$
\mathscr{H}\left(A_{1}, A_{2}\right)=\max \left\{e\left(A_{1}, A_{2}\right), e\left(A_{2}, A_{1}\right)\right\},
$$

where $e\left(A_{1}, A_{2}\right)=\sup _{a \in A_{1}} d\left(a, A_{2}\right)$ with $d\left(a, A_{2}\right)=\inf _{b \in A_{2}}\|a-b\|$. Note that [25] if $A_{1}$ and $A_{2}$ are compact subsets in $X$, then for each $a \in A_{1}$ there exists $b \in A_{2}$ such that

$$
\|a-b\| \leq \mathscr{H}\left(A_{1}, A_{2}\right) .
$$

Definition 2.3. [see [32]] Let $\mathscr{H}(\cdot, \cdot)$ be the Hausdorff metric on the collection $C B\left(X^{*}\right)$ of all nonempty, closed and bounded subsets of $X^{*}$, which is defined by

$$
\mathscr{H}(A, B)=\max \{e(A, B), e(B, A)\}
$$

for $A$ and $B$ in $C B\left(X^{*}\right)$. A nonempty set-valued mapping $F: X \rightarrow C B\left(X^{*}\right)$ is said to be 
(i) $\mathscr{H}$-hemicontinuous, if for any $x, y \in X$, the function $t \mapsto \mathscr{H}(F(x+t(y-x)), F(x))$ from $[0,1]$ into $\mathbf{R}^{+}=[0,+\infty)$ is continuous at $0^{+}$;

(ii) $\mathscr{H}$-continuous, if for any $\epsilon>0$ and any fixed $x \in X$, there exists $\delta>0$ such that for all $y \in X$ with $\|y-x\|<\delta$, one has $\mathscr{H}(F(y), F(x))<\epsilon$.

Remark 2.2. Clearly, the $\mathscr{H}$-continuity implies the $\mathscr{H}$-hemicontinuity, but the converse is not true in general.

Theorem 2.1 (see [7]). Let $C \subset X$ be nonempty, closed and convex, $C^{*} \subset X^{*}$ be nonempty, closed, convex and bounded, $\varphi: X \rightarrow \mathbf{R}$ be proper, convex and lower semicontinuous and $y \in C$ be arbitrary. Assume that, for each $x \in C$, there exists $x^{*}(x) \in C^{*}$ such that

$$
\left\langle x^{*}(x), x-y\right\rangle \geq \varphi(y)-\varphi(x) .
$$

Then, there exists $y^{*} \in C^{*}$ such that

$$
\left\langle y^{*}, x-y\right\rangle \geq \varphi(y)-\varphi(x), \quad \forall x \in C .
$$

Definition 2.4 (see [35]). Let $S$ be a nonempty subset of $X$. The measure of noncompactness $\mu$ of the set $S$ is defined by

$$
\mu(S):=\inf \left\{\epsilon>0: S \subset \bigcup_{i=1}^{n} S_{i}, \operatorname{diam}\left(S_{i}\right)<\epsilon, i=1,2, \ldots, n\right\},
$$

where $\operatorname{diam}\left(S_{i}\right)$ means the diameter of set $S_{i}$.

\section{Well-posedness of GVHVI with metric characterizations}

Based on the concepts of well-posedness in [26, 27, 31, 32, 33, 34], we introduce some concepts of well-posedness for the generalized variational-hemivariational inequality GVHVI with perturbation, establish its metric characterizations and derive some conditions under which the generalized variational-hemivariational inequality GVHVI is strongly $\alpha$-well-posed in the generalized sense in Euclidean space $\mathbf{R}^{\mathbf{n}}$. Let $\alpha: X \rightarrow \mathbf{R}^{+}=[0,+\infty)$ be a convex and continuous functional with $\alpha(t x)=t \alpha(x) \forall t \geq 0$ and $\forall x \in X$.

Definition 3.1. A sequence $\left\{x_{n}\right\} \subset X$ is said to be an $\alpha$-approximating sequence for GVHVI if there exist $u_{n} \in F\left(x_{n}\right), n \in \mathbf{N}$ and a nonnegative sequence $\left\{\epsilon_{n}\right\}$ with $\epsilon_{n} \rightarrow 0$ as $n \rightarrow \infty$ such that

$$
\left\langle u_{n}+T x_{n}-f, y-x_{n}\right\rangle+J^{\circ}\left(x_{n}, y-x_{n}\right)+G(y)-G\left(x_{n}\right) \geq-\epsilon_{n} \alpha\left(y-x_{n}\right), \quad \forall y \in X, n \in \mathbf{N} .
$$

In particular, if $\alpha(\cdot)=\|\cdot\|$ the norm of $X$, then $\left\{x_{n}\right\}$ is said to be an approximating sequence for GVHVI. 
Definition 3.2. GVHVI is said to be strongly (resp., weakly) $\alpha$-well-posed if GVHVI has a unique solution in $X$ and every $\alpha$-approximating sequence converges strongly (resp., weakly) to the unique solution. In particular, if $\alpha(\cdot)=\|\cdot\|$ the norm of $X$, then GVHVI is said to be strongly (resp., weakly) well-posed.

Remark 3.1. The strong $\alpha$-well-posedness implies the weak $\alpha$-well-posedness, but the converse is not true in general.

Definition 3.3. GVHVI is said to be strongly (resp., weakly) $\alpha$-well-posed in the generalized sense if GVHVI has a nonempty solution set $S$ in $X$ and every $\alpha$-approximating sequence has a subsequence which converges strongly (resp., weakly) to some point of solution set $S$.

Remark 3.2. The strong $\alpha$-well-posedness in the generalized sense implies the weak $\alpha$-wellposedness in the generalized sense, but the converse is not true in general.

Remark 3.3. The concepts of strong and weak $\alpha$-well-posedness for GVHVI introduced in this paper include as special cases Definitions 3.2-3.3 in Xiao and Huang [26].

For any $\epsilon>0$, we define the following two sets:

$$
\begin{aligned}
\Omega_{\alpha}(\epsilon) & =\left\{x \in X: \exists u \in F(x) \text { s.t. }\langle u+T x-f, y-x\rangle+J^{\circ}(x, y-x)+G(y)-G(x)\right. \\
& \geq-\epsilon \alpha(y-x), \forall y \in X\}
\end{aligned}
$$

and

$$
\begin{aligned}
\Delta_{\alpha}(\epsilon) & =\left\{x \in X:\langle v+T x-f, y-x\rangle+J^{\circ}(x, y-x)+G(y)-G(x)\right. \\
& \geq-\epsilon \alpha(y-x), \forall y \in X, v \in F(y)\} .
\end{aligned}
$$

Lemma 3.1. Suppose that $F: X \rightarrow 2^{X^{*}}$ is a nonempty compact-valued mapping which is $\mathscr{H}$ hemicontinuous and monotone. Let $G: X \rightarrow \mathbf{R} \cup\{+\infty\}$ be a proper, convex and lower semicontinuous functional. Then, $\Omega_{\alpha}(\epsilon)=\Delta_{\alpha}(\epsilon)$, for all $\epsilon>0$.

Proof. We first claim that $\Omega_{\alpha}(\epsilon) \subset \Delta_{\alpha}(\epsilon)$. Indeed, take an arbitrary $x \in \Omega_{\alpha}(\epsilon)$. Then there exists $u \in F(x)$ such that

$$
\langle u+T x-f, y-x\rangle+J^{\circ}(x, y-x)+G(y)-G(x) \geq-\epsilon \alpha(y-x), \quad \forall y \in X .
$$

So, it follows from the monotonicity of the mapping $F$ that

$$
\begin{aligned}
& \langle v+T x-f, y-x\rangle+J^{\circ}(x, y-x)+G(y)-G(x) \\
& \geq\langle u+T x-f, y-x\rangle+J^{\circ}(x, y-x)+G(y)-G(x) \\
& \geq-\epsilon \alpha(y-x), \forall y \in X, v \in F(y) .
\end{aligned}
$$

This means that $x \in \Delta_{\alpha}(\epsilon)$. Thus, $\Omega_{\alpha}(\epsilon) \subset \Delta_{\alpha}(\epsilon)$. 
Now we show that $\Delta_{\alpha}(\epsilon) \subset \Omega_{\alpha}(\epsilon)$. Indeed, for any $x \in \Delta_{\alpha}(\epsilon)$, we have

$$
\langle v+T x-f, y-x\rangle+J^{\circ}(x, y-x)+G(y)-G(x) \geq-\epsilon \alpha(y-x), \quad \forall y \in X, v \in F(y) .
$$

Given any $y \in X$ we define $y_{t}=t y+(1-t) x=x+t(y-x)$ for all $t \in(0,1)$. Replacing $y$ and $v$ by $y_{t}$ and $v_{t}$ in the last inequality, respectively, we deduce from the convexity of $G$ and the positive homogeneousness of the functions $y \mapsto J^{\circ}(x, y)$ and $\alpha$ that for each $v_{t} \in F\left(y_{t}\right)$,

$$
\begin{aligned}
-t \epsilon \alpha(y-x) & =-\epsilon \alpha(t(y-x)) \\
& \leq\left\langle v_{t}+T x-f, t(y-x)\right\rangle+J^{\circ}(x, t(y-x))+G\left(y_{t}\right)-G(x) \\
& \leq\left\langle v_{t}+T x-f, t(y-x)\right\rangle+J^{\circ}(x, t(y-x))+t(G(y)-G(x)) \\
& =t\left[\left\langle v_{t}+T x-f, y-x\right\rangle+J^{\circ}(x, y-x)+G(y)-G(x)\right],
\end{aligned}
$$

which hence implies that for each $t \in(0,1)$ and each $v_{t} \in F\left(y_{t}\right)$,

$$
\left\langle v_{t}+T x-f, y-x\right\rangle+J^{\circ}(x, y-x)+G(y)-G(x) \geq-\epsilon \alpha(y-x) .
$$

Since $F: X \rightarrow 2^{X^{*}}$ is a nonempty compact-valued mapping, $F\left(y_{t}\right)$ and $F(x)$ are nonempty compact sets. Hence, by Nadler's result [25] we know that for each $t \in(0,1)$ and each fixed $v_{t} \in F\left(y_{t}\right)$ there exists an $u_{t} \in F(x)$ such that $\left\|v_{t}-u_{t}\right\| \leq \mathscr{H}\left(F\left(y_{t}\right), F(x)\right)$. Since $F(x)$ is compact, without loss of generality we may assume that $u_{t} \rightarrow u \in F(x)$ as $t \rightarrow 0^{+}$. Since $F$ is $\mathscr{H}$-hemicontinuous, we obtain that

$$
\left\|v_{t}-u_{t}\right\| \leq \mathscr{H}\left(F\left(y_{t}\right), F(x)\right) \rightarrow 0 \quad \text { as } t \rightarrow 0^{+}
$$

which immediately leads to

$$
\left\|v_{t}-u\right\| \leq\left\|v_{t}-u_{t}\right\|+\left\|u_{t}-u\right\| \rightarrow 0 \quad \text { as } t \rightarrow 0^{+} .
$$

Hence, taking the limsup as $t \rightarrow 0^{+}$in (3.2) we conclude from (3.3) that

$$
\langle u+T x-f, y-x\rangle+J^{\circ}(x, y-x)+G(y)-G(x) \geq-\epsilon \alpha(y-x) .
$$

By the arbitrariness of $y \in X$, we know that $x \in \Omega_{\alpha}(\epsilon)$, which implies that $\Delta_{\alpha}(\epsilon) \subset \Omega_{\alpha}(\epsilon)$. This completes the proof.

Lemma 3.2. Suppose that $T: X \rightarrow X^{*}$ is continuous and let $G: X \rightarrow \mathbf{R} \cup\{+\infty\}$ be a proper, convex and lower semicontinuous functional. Then, $\Delta_{\alpha}(\epsilon)$ is closed in $X$ for all $\epsilon>0$.

Proof. Let $\left\{x_{n}\right\} \subset \Delta_{\alpha}(\epsilon)$ be a sequence such that $x_{n} \rightarrow x$ in $X$. Then

$$
\left\langle v+T x_{n}-f, y-x_{n}\right\rangle+J^{\circ}\left(x_{n}, y-x_{n}\right)+G(y)-G\left(x_{n}\right) \geq-\epsilon \alpha\left(y-x_{n}\right), \quad \forall y \in X, v \in F(y) .
$$


Since $T: X \rightarrow X^{*}$ is continuous, $\alpha: X \rightarrow[0,+\infty)$ is continuous, Clarke's generalized directional derivative $J^{\circ}(x, y)$ is upper semicontinuous with respect to $(x, y)$ and $G: X \rightarrow \mathbf{R} \cup\{+\infty\}$ is lower semicontinuous, we have that $\left\langle T x_{n}-f, y-x_{n}\right\rangle \rightarrow\langle T x-f, y-x\rangle, \alpha\left(y-x_{n}\right) \rightarrow \alpha(y-x)$,

$$
\limsup _{n \rightarrow \infty} J^{\circ}\left(x_{n}, y-x_{n}\right) \leq J^{\circ}(x, y-x) \text { and } \limsup _{n \rightarrow \infty}-G\left(x_{n}\right) \leq-G(x) .
$$

So, taking the limsup as $n \rightarrow \infty$ at both sides of (3.4), we have

$$
\langle v+T x-f, y-x\rangle+J^{\circ}(x, y-x)+G(y)-G(x) \geq-\epsilon \alpha(y-x), \quad \forall y \in X, v \in F(y),
$$

which implies that $x \in \Delta_{\alpha}(\epsilon)$. Thus, $\Delta_{\alpha}(\epsilon)$ is closed in $X$. This completes the proof.

Corollary 3.1. Suppose that $F: X \rightarrow 2^{X^{*}}$ is a nonempty compact-valued mapping which is $\mathscr{H}$-hemicontinuous and monotone. Let $T: X \rightarrow X^{*}$ be a continuous mapping and $G: X \rightarrow$ $\mathbf{R} \cup\{+\infty\}$ be a proper, convex and lower semicontinuous functional. Then, for all $\epsilon>0, \Omega_{\alpha}(\epsilon)=$ $\Delta_{\alpha}(\epsilon)$ is closed in $X$.

Theorem 3.1. Suppose that $F: X \rightarrow 2^{X^{*}}$ is a nonempty compact-valued mapping which is $\mathscr{H}$-hemicontinuous and monotone. Let $T: X \rightarrow X^{*}$ be a continuous mapping and $G: X \rightarrow$ $\mathbf{R} \cup\{+\infty\}$ be a proper, convex and lower semicontinuous functional. Then, GVHVI is strongly $\alpha$-well-posed if and only if

$$
\Omega_{\alpha}(\epsilon) \neq \varnothing \quad \forall \epsilon>0 \text { and } \operatorname{diam}\left(\Omega_{\alpha}(\epsilon)\right) \rightarrow 0 \text { as } \epsilon \rightarrow 0 .
$$

Proof. (Necessity) Suppose that GVHVI is strongly $\alpha$-well-posed. Then, GVHVI has a unique solution which belongs to $\Omega_{\alpha}(\epsilon)$ and so $\Omega_{\alpha}(\epsilon) \neq \varnothing$ for all $\epsilon>0$. If $\operatorname{diam}\left(\Omega_{\alpha}(\epsilon)\right)$ does not converge to 0 as $\epsilon \rightarrow 0$, then there exist a constant $l>0$, a nonnegative sequence $\left\{\epsilon_{n}\right\}$ with $\epsilon_{n} \rightarrow 0$ and $x_{n}, y_{n} \in \Omega_{\alpha}\left(\epsilon_{n}\right)$ such that

$$
\left\|x_{n}-y_{n}\right\|>l, \quad \forall n \in \mathbf{N} .
$$

Since $x_{n}, y_{n} \in \Omega_{\alpha}\left(\epsilon_{n}\right)$, we know that $\left\{x_{n}\right\}$ and $\left\{y_{n}\right\}$ are both $\alpha$-approximating sequences for GVHVI. It follows from strong $\alpha$-well-posedness of GVHVI that both $\left\{x_{n}\right\}$ and $\left\{y_{n}\right\}$ converge strongly to the unique solution of GVHVI, which is a contradiction to (3.6).

(Sufficiency) Let $\left\{x_{n}\right\} \subset X$ be an $\alpha$-approximating sequence for GVHVI. Then, there exist $u_{n} \in$ $F\left(x_{n}\right), n \in \mathbf{N}$ and a nonnegative sequence $\left\{\epsilon_{n}\right\}$ with $\epsilon_{n} \rightarrow 0$ such that

$$
\left\langle u_{n}+T x_{n}-f, y-x_{n}\right\rangle+J^{\circ}\left(x_{n}, y-x_{n}\right)+G(y)-G\left(x_{n}\right) \geq-\epsilon_{n} \alpha\left(y-x_{n}\right), \quad \forall y \in X, n \in \mathbf{N},
$$

which implies that $x_{n} \in \Omega_{\alpha}\left(\epsilon_{n}\right)$. From (3.5), It follows that $\left\{x_{n}\right\}$ is a Cauchy sequence, and so $\left\{x_{n}\right\}$ converges strongly to some point $x \in X$. Since the mapping $F$ is monotone, the mapping $T$ is continuous, the Clarke's generalized directional derivative $J^{\circ}(x, y)$ is upper semicontinuous with respect to $(x, y)$ and $G$ is lower semicontinuous, we deduce from (3.7) and the property of the functional $\alpha$ that for all $y \in X$ and $v \in F(y)$

$$
\langle v+T x-f, y-x\rangle+J^{\circ}(x, y-x)+G(y)-G(x)
$$




$$
\begin{aligned}
& \geq \limsup _{n \rightarrow \infty}\left\{\left\langle v+T x_{n}-f, y-x_{n}\right\rangle+J^{\circ}\left(x_{n}, y-x_{n}\right)+G(y)-G\left(x_{n}\right)\right\} \\
& \geq \limsup _{n \rightarrow \infty}\left\{\left\langle u_{n}+T x_{n}-f, y-x_{n}\right\rangle+J^{\circ}\left(x_{n}, y-x_{n}\right)+G(y)-G\left(x_{n}\right)\right\} \\
& \geq \limsup _{n \rightarrow \infty}-\epsilon_{n} \alpha\left(y-x_{n}\right) \\
& =\limsup _{n \rightarrow \infty}-\alpha\left(\epsilon_{n}\left(y-x_{n}\right)\right) \\
& =-\alpha(0)=0 .
\end{aligned}
$$

Given any $y \in X$ we define $y_{t}=x+t(y-x)$ for all $t \in(0,1)$. Replacing $y$ and $v$ by $y_{t}$ and $v_{t}$ in (3.8), we obtain from the positive homogeneousness of $J^{\circ}(x, y)$ with respect to $y$ and the convexity of $G$ that for each $v_{t} \in F\left(y_{t}\right)$,

$$
\left\langle v_{t}+T x-f, y-x\right\rangle+J^{\circ}(x, y-x)+G(y)-G(x) \geq 0 .
$$

Since $F: X \rightarrow 2^{X^{*}}$ is a nonempty compact-valued mapping, $F\left(y_{t}\right)$ and $F(x)$ are nonempty compact sets. Hence, by Nadler's result [25] we know that for each $t \in(0,1)$ and each fixed $v_{t} \in F\left(y_{t}\right)$ there exists an $u_{t} \in F(x)$ such that $\left\|v_{t}-u_{t}\right\| \leq \mathscr{H}\left(F\left(y_{t}\right), F(x)\right)$. Since $F(x)$ is compact, without loss of generality we may assume that $u_{t} \rightarrow u \in F(x)$ as $t \rightarrow 0^{+}$. Since $F$ is $\mathscr{H}$-hemicontinuous, we obtain that

$$
\left\|v_{t}-u_{t}\right\| \leq \mathscr{H}\left(F\left(y_{t}\right), F(x)\right) \rightarrow 0 \quad \text { as } t \rightarrow 0^{+},
$$

which immediately leads to

$$
\left\|v_{t}-u\right\| \leq\left\|v_{t}-u_{t}\right\|+\left\|u_{t}-u\right\| \rightarrow 0 \quad \text { as } t \rightarrow 0^{+} .
$$

Now, taking the limsup as $t \rightarrow 0^{+}$in (3.9), we get

$$
\langle u+T x-f, y-x\rangle+J^{\circ}(x, y-x)+G(y)-G(x) \geq 0, \quad \forall y \in X,
$$

which implies that $x$ solves GVHVI.

To complete the proof of Theorem 3.1, we need only to prove that GVHVI has a unique solution. Assume by contradiction that GVHVI has two distinct solutions $x_{1}$ and $x_{2}$. Then it is easy to see that $x_{1}, x_{2} \in \Omega_{\alpha}(\epsilon)$ for all $\epsilon>0$ and

$$
0<\left\|x_{1}-x_{2}\right\| \leq \operatorname{diam}\left(\Omega_{\alpha}(\epsilon)\right) \rightarrow 0,
$$

which is a contradiction. Therefore, GVHVI has a unique solution.

This completes the proof.

Theorem 3.2. Suppose that $F: X \rightarrow 2^{X^{*}}$ is a nonempty compact-valued mapping which is $\mathscr{H}$-hemicontinuous and monotone. Let $T: X \rightarrow X^{*}$ be a continuous mapping and $G: X \rightarrow$ $\mathbf{R} \cup\{+\infty\}$ be a proper, convex and lower semicontinuous functional. Then, GVHVI is strongly $\alpha$-well-posed in the generalized sense if and only if

$$
\Omega_{\alpha}(\epsilon) \neq \varnothing \forall \epsilon>0 \text { and } \mu\left(\Omega_{\alpha}(\epsilon)\right) \rightarrow 0 \text { as } \epsilon \rightarrow 0 .
$$


Proof. (Necessity) Suppose that GVHVI is strongly $\alpha$-well-posed in the generalized sense. Then the solution set $S$ of GVHVI is nonempty and $S \subset \Omega_{\alpha}(\epsilon)$ for any $\epsilon>0$. Furthermore, the solution set $S$ of GVHVI is also compact. As a matter of fact, for any sequence $\left\{x_{n}\right\} \subset S$, it follows from $S \subset \Omega_{\alpha}(\epsilon)$ for any $\epsilon>0$ that $\left\{x_{n}\right\} \subset S$ is an $\alpha$-approximating sequence for GVHVI. Since GVHVI is strongly $\alpha$-well-posed in the generalized sense, $\left\{x_{n}\right\}$ has a subsequence which converges strongly to some point of the solution set $S$. Thus, the solution set $S$ of GVHVI is compact. Now we show that $\mu\left(\Omega_{\alpha}(\epsilon)\right) \rightarrow 0$ as $\epsilon \rightarrow 0$. From $S \subset \Omega_{\alpha}(\epsilon)$ for any $\epsilon>0$, we get

$$
\mathscr{H}\left(\Omega_{\alpha}(\epsilon), S\right)=\max \left\{e\left(\Omega_{\alpha}(\epsilon), S\right), e\left(S, \Omega_{\alpha}(\epsilon)\right)\right\}=e\left(\Omega_{\alpha}(\epsilon), S\right) .
$$

Taking into account the compactness of the solution set $S$, we obtain from (3.11) that

$$
\mu\left(\Omega_{\alpha}(\epsilon)\right) \leq 2 \mathscr{H}\left(\Omega_{\alpha}(\epsilon), S\right)=2 e\left(\Omega_{\alpha}(\epsilon), S\right) .
$$

Thus, to prove $\mu\left(\Omega_{\alpha}(\epsilon)\right) \rightarrow 0$ as $\epsilon \rightarrow 0$, it suffices to show that $e\left(\Omega_{\alpha}(\epsilon), S\right) \rightarrow 0$ as $\epsilon \rightarrow 0$. Assume by contradiction that $e\left(\Omega_{\alpha}(\epsilon), S\right) \nrightarrow 0$ as $\epsilon \rightarrow 0$. Then there exist a constant $l>0$, a sequence $\left\{\epsilon_{n}\right\} \subset[0, \infty)$ with $\epsilon_{n} \rightarrow 0$ and $x_{n} \in \Omega_{\alpha}\left(\epsilon_{n}\right)$ such that

$$
x_{n} \notin S+B(0, l)
$$

where $B(0, l)$ is the closed ball centered at 0 with radius $l$. Since $\left\{x_{n}\right\}$ is an $\alpha$-approximating sequence for GVHVI and GVHVI is strongly $\alpha$-well-posed in the generalized sense, there exists a subsequence $\left\{x_{n_{k}}\right\}$ converging strongly to some point $x \in S$, which is a contradiction to (3.12). Consequently, $\mu\left(\Omega_{\alpha}(\epsilon)\right) \rightarrow 0$ as $\epsilon \rightarrow 0$.

(Sufficiency) Assume that condition (3.10) holds. By Corollary 3.1, we obtain that $\Omega_{\alpha}(\epsilon)$ is nonempty and closed for all $\epsilon>0$. Observe that

$$
S=\bigcap_{\epsilon>0} \Omega_{\alpha}(\epsilon)
$$

Since $\mu\left(\Omega_{\alpha}(\epsilon)\right) \rightarrow 0$ as $\epsilon \rightarrow 0$, by applying the theorem in [35, p.412], one easily concludes that $S$ is nonempty and compact with

$$
e\left(\Omega_{\alpha}(\epsilon), S\right)=\mathscr{H}\left(\Omega_{\alpha}(\epsilon), S\right) \rightarrow 0 \quad \text { as } \epsilon \rightarrow 0 .
$$

Let $\left\{x_{n}\right\} \subset X$ be an $\alpha$-approximating sequence for GVHVI. Then there exist $u_{n} \in F\left(x_{n}\right), n \in \mathbf{N}$ and a nonnegative sequence $\left\{\epsilon_{n}\right\}$ with $\epsilon_{n} \rightarrow 0$ such that

$$
\left\langle u_{n}+T x_{n}-f, y-x_{n}\right\rangle+J^{\circ}\left(x_{n}, y-x_{n}\right)+G(y)-G\left(x_{n}\right) \geq-\epsilon_{n} \alpha\left(y-x_{n}\right), \quad \forall y \in X, n \in \mathbf{N},
$$

and so $x_{n} \in \Omega_{\alpha}\left(\epsilon_{n}\right)$ by the definition of $\Omega_{\alpha}\left(\epsilon_{n}\right)$. It follows from (3.14) that

$$
d\left(x_{n}, S\right) \leq e\left(\Omega_{\alpha}\left(\epsilon_{n}\right), S\right) \rightarrow 0 .
$$


Since the solution set $S$ is compact, there exists $\bar{x}_{n} \in S$ such that

$$
\left\|x_{n}-\bar{x}_{n}\right\|=d\left(x_{n}, S\right) \rightarrow 0
$$

Again from the compactness of the solution set $S,\left\{\bar{x}_{n}\right\}$ has a subsequence $\left\{\bar{x}_{n_{k}}\right\}$ converging strongly to some $\bar{x} \in S$. It follows from (3.15) that

$$
\left\|x_{n_{k}}-\bar{x}\right\| \leq\left\|x_{n_{k}}-\bar{x}_{n_{k}}\right\|+\left\|\bar{x}_{n_{k}}-\bar{x}\right\| \rightarrow 0,
$$

which implies that $\left\{x_{n_{k}}\right\}$ converges strongly to $\bar{x}$. Therefore, GVHVI is strongly $\alpha$-well-posed in the generalized sense. This completes the proof.

The following theorem gives some conditions under which the generalized variationalhemivariational inequality is strongly $\alpha$-well-posed in the generalized sense in Euclidean space $\mathbf{R}^{\mathbf{n}}$.

Theorem 3.3. Let $F: \mathbf{R}^{\mathbf{n}} \rightarrow C B\left(\mathbf{R}^{\mathbf{n}}\right)$ be a nonempty $\not{H}$-hemicontinuous and monotone multifunction. Let $T: \mathbf{R}^{\mathbf{n}} \rightarrow \mathbf{R}^{\mathbf{n}}$ be a continuous mapping and $G: \mathbf{R}^{\mathbf{n}} \rightarrow \mathbf{R} \cup\{+\infty\}$ be a proper, convex and lower semicontinuous functional. If there exists some $\epsilon>0$ such that $\Omega_{\alpha}(\epsilon)$ is nonempty and bounded, then generalized variational-hemivariational inequality GVHVI is strongly $\alpha$ well-posed in the generalized sense.

Proof. Suppose that $\left\{x_{n}\right\}$ is an $\alpha$-approximating sequence for GVHVI. Then, there exist $u_{n} \in$ $F\left(x_{n}\right), n \in \mathbf{N}$ and a nonnegative sequence $\left\{\epsilon_{n}\right\}$ with $\epsilon_{n} \rightarrow 0$ as $n \rightarrow \infty$ such that

$$
\left\langle u_{n}+T x_{n}-f, y-x_{n}\right\rangle+J^{\circ}\left(x_{n}, y-x_{n}\right)+G(y)-G\left(x_{n}\right) \geq-\epsilon_{n} \alpha\left(y-x_{n}\right), \quad \forall y \in \mathbf{R}^{\mathbf{n}}, n \in \mathbf{N} .
$$

Let $\epsilon_{0}>0$ be such that $\Omega_{\alpha}\left(\epsilon_{0}\right)$ is nonempty and bounded. Then, there exists $n_{0}$ such that $x_{n} \in$ $\Omega_{\alpha}\left(\epsilon_{0}\right)$ for all $n \geq n_{0}$. So, it follows that $\left\{x_{n}\right\}$ is bounded in $\mathbf{R}^{\mathbf{n}}$ by the boundedness of $\Omega_{\alpha}\left(\epsilon_{0}\right)$. Thus, there exists a subsequence $\left\{x_{n_{k}}\right\}$ such that $x_{n_{k}} \rightarrow \bar{x}$ as $k \rightarrow \infty$. Since the mapping $F$ is monotone, the mapping $T$ is continuous, Clarke's generalized directional derivative $J^{\circ}(x, y)$ is upper semicontinuous with respect to $(x, y)$ and $G$ is lower semicontinuous, it follows from (3.16) and the property of the functional $\alpha$ that for any $y \in \mathbf{R}^{\mathbf{n}}, v \in F(y)$,

$$
\begin{aligned}
\langle v & +T \bar{x}-f, y-\bar{x}\rangle+J^{\circ}(\bar{x}, y-\bar{x})+G(y)-G(\bar{x}) \\
& \geq \limsup _{k \rightarrow \infty}\left\{\left\langle v+T x_{n_{k}}-f, y-x_{n_{k}}\right\rangle+J^{\circ}\left(x_{n_{k}}, y-x_{n_{k}}\right)+G(y)-G\left(x_{n_{k}}\right)\right\} \\
& \geq \limsup _{k \rightarrow \infty}\left\{\left\langle u_{n_{k}}+T x_{n_{k}}-f, y-x_{n_{k}}\right\rangle+J^{\circ}\left(x_{n_{k}}, y-x_{n_{k}}\right)+G(y)-G\left(x_{n_{k}}\right)\right\} \\
& \geq \limsup _{k \rightarrow \infty}-\epsilon_{n_{k}} \alpha\left(y-x_{n_{k}}\right) \\
& =\limsup _{k \rightarrow \infty}-\alpha\left(\epsilon_{n_{k}}\left(y-x_{n_{k}}\right)\right)
\end{aligned}
$$




$$
=-\alpha(0)=0 .
$$

Given any $y \in \mathbf{R}^{\mathbf{n}}$ we define $y_{t}=t y+(1-t) \bar{x}=\bar{x}+t(y-\bar{x})$ for all $t \in(0,1)$. Replacing $y$ and $v$ in (3.17) by $y_{t}$ and $v_{t}$, respectively, we deduce from the positive homogeneousness of the function $y \mapsto J^{\circ}(x, y)$ and the convexity of $G$ that for each $v_{t} \in F\left(y_{t}\right)$

$$
\left\langle v_{t}+T \bar{x}-f, y-\bar{x}\right\rangle+J^{\circ}(\bar{x}, y-\bar{x})+G(y)-G(\bar{x}) \geq 0 .
$$

Since $F: \mathbf{R}^{\mathbf{n}} \rightarrow C B\left(\mathbf{R}^{\mathbf{n}}\right)$ is a nonempty compact-valued mapping, $F\left(y_{t}\right)$ and $F(\bar{x})$ are nonempty compact sets. Hence, by Nadler's result [25] we know that for each $t \in(0,1)$ and each fixed $v_{t} \in F\left(y_{t}\right)$ there exists an $u_{t} \in F(\bar{x})$ such that $\left\|v_{t}-u_{t}\right\| \leq \mathscr{H}\left(F\left(y_{t}\right), F(\bar{x})\right)$. Since $F(\bar{x})$ is compact, without loss of generality we may assume that $u_{t} \rightarrow \bar{u} \in F(\bar{x})$ as $t \rightarrow 0^{+}$. Since $F$ is $\mathscr{H}$-hemicontinuous, we obtain that

$$
\left\|v_{t}-u_{t}\right\| \leq \mathscr{H}\left(F\left(y_{t}\right), F(\bar{x})\right) \rightarrow 0 \quad \text { as } t \rightarrow 0^{+},
$$

which immediately leads to

$$
\left\|v_{t}-\bar{u}\right\| \leq\left\|v_{t}-u_{t}\right\|+\left\|u_{t}-\bar{u}\right\| \rightarrow 0 \quad \text { as } t \rightarrow 0^{+} .
$$

Now, taking the limsup as $t \rightarrow 0^{+}$in (3.18), we get

$$
\langle\bar{u}+T \bar{x}-f, y-\bar{x}\rangle+J^{\circ}(\bar{x}, y-\bar{x})+G(y)-G(\bar{x}) \geq 0, \quad \forall y \in \mathbf{R}^{\mathbf{n}},
$$

which implies that $\bar{x}$ solves GVHVI. Therefore, GVHVI is strongly $\alpha$-well-posed in the generalized sense. This completes the proof.

Remark 3.4. Whenever the mapping $F$ is single-valued, GVHVI (1.1) reduces to VHVI (1) in [26]. In this case, it is easy to see that the above Lemmas 3.1-3.2, Corollary 3.1 and Theorems $3.1-3.3$ reduce to Lemmas 3.1-3.2, Corollary 3.1 and Theorems 3.1-3.3 in [26], respectively. Therefore, the above Lemmas 3.1-3.2, Corollary 3.1 and Theorems 3.1-3.3 improve, extend and develop Lemmas 3.1-3.2, Corollary 3.1 and Theorems 3.1-3.3 in [26], respectively.

\section{Links with Well-Posedness for the Corresponding Inclusion Problem}

In this section, we introduce the concept of $\alpha$-well-posedness for the inclusion problem and investigate the relations between the $\alpha$-well-posedness of generalized variationalhemivariational inequality GVHVI and the $\alpha$-well-posedness of the corresponding inclusion problem. In what follows, we always assume that $\Gamma$ is a nonempty set-valued mapping from a real reflexive Banach space $X$ to its dual space $X^{*}$. The inclusion problem associated with the mapping $\Gamma$ is defined by

$\operatorname{IP}(\Gamma)$ : find $x \in X$ such that $0 \in \Gamma(x)$. 
Definition 4.1. A sequence $\left\{x_{n}\right\} \subset X$ is called an $\alpha$-approximating sequence for the inclusion problem $\mathrm{IP}(\Gamma)$ if there exist $w_{n} \in \Gamma\left(x_{n}\right), n \in \mathbf{N}$ and a nonnegative sequence $\left\{\epsilon_{n}\right\}$ with $\left\|w_{n}\right\|+$ $\epsilon_{n} \rightarrow 0$ as $n \rightarrow \infty$, such that

$$
\left\langle w_{n}, y-x_{n}\right\rangle \geq-\epsilon_{n} \alpha\left(y-x_{n}\right), \quad \forall y \in X, n \in \mathbf{N} .
$$

Definition 4.2. We say that the inclusion problem $\operatorname{IP}(\Gamma)$ is strongly (resp., weakly) $\alpha$-wellposed if it has a unique solution and every $\alpha$-approximating sequence converges strongly (resp., weakly) to the unique solution of IP( $\Gamma)$.

Definition 4.3. We say that the inclusion problem $\mathrm{IP}(\Gamma)$ is strongly (resp., weakly) $\alpha$ well-posed in the generalized sense if the solution set $S$ of $\operatorname{IP}(\Gamma)$ is nonempty and every $\alpha$-approximating sequence has a subsequence which converges strongly (resp., weakly) to some point of the solution set $S$ for $\operatorname{IP}(\Gamma)$.

The following two theorems establish the relations between the strong (resp., weak) $\alpha$ well-posedness of generalized variational-hemivariational inequality GVHVI and the strong (resp., weak) $\alpha$-well-posedness of the corresponding inclusion problem.

Theorem 4.1. Let $F: X \rightarrow 2^{X^{*}}$ be a nonempty set-valued mapping from a Banach space $X$ to its dual $X^{*}$, and $T: X \rightarrow X^{*}$ be a mapping from $X$ to $X^{*}, J: X \rightarrow \mathbf{R}$ be a locally Lipschitz functional and $G: X \rightarrow \mathbf{R} \cup\{+\infty\}$ be a proper, convex and lower semicontinuous functional. The generalized variational-hemivariational inequality GVHVI is strongly (resp., weakly) $\alpha$ well-posed if and only if the corresponding inclusion problem $\operatorname{IP}(F+T-f+\bar{\partial} J+\partial G)$ is strongly (resp., weakly) $\alpha$-well-posed.

Theorem 4.2. Let $F: X \rightarrow 2^{X^{*}}$ be a nonempty set-valued mapping from a Banach space $X$ to its dual $X^{*}$, and $T: X \rightarrow X^{*}$ be a mapping from $X$ to $X^{*}, J: X \rightarrow \mathbf{R}$ be a locally Lipschitz functional and $G: X \rightarrow \mathbf{R} \cup\{+\infty\}$ be a proper, convex and lower semicontinuous functional. The generalized variational-hemivariational inequality GVHVI is strongly (resp., weakly) $\alpha$ well-posed in the generalized sense if and only if the corresponding inclusion problem $\operatorname{IP}(F+$ $T-f+\bar{\partial} J+\partial G)$ is strongly (resp., weakly) $\alpha$-well-posed in the generalized sense.

In order to prove Theorems 4.1 and 4.2 , we need the following lemma.

Lemma 4.3. Let $F: X \rightarrow 2^{X^{*}}$ be a nonempty set-valued mapping from a Banach space $X$ to its dual $X^{*}$, and $T: X \rightarrow X^{*}$ be a mapping from $X$ to $X^{*}, J: X \rightarrow \mathbf{R}$ be a locally Lipschitz functional and $G: X \rightarrow \mathbf{R} \cup\{+\infty\}$ be a proper, convex and lower semicontinuous functional. Then the following two statements are equivalent:

(i) $x \in X$ is a solution to the generalized variational-hemivariational inequality GVHVI; 
(ii) $x$ is a solution to the inclusion problem

$$
\begin{aligned}
& \operatorname{IP}(F+T-f+\bar{\partial} J+\partial G): \text { Find } x \in X \text { such that } \\
& \qquad 0 \in(F+T-f+\bar{\partial} J+\partial G) x .
\end{aligned}
$$

Proof. We first claim that (ii) $\Rightarrow$ (i). Indeed, let $x \in X$ be a solution to the inclusion problem $\operatorname{IP}(F+T-f+\bar{\partial} J+\partial G)$. Then, there exist $u \in F(x), \xi \in \bar{\partial} J(x)$ and $\varrho \in \partial G(x)$ such that

$$
0=u+T x-f+\xi+\varrho
$$

By multiplying $y-x$ at both sides of the last equality, we obtain, from the definitions of the Clarke's generalized gradient for locally Lipschitz functional and the subgradient for convex functional, that

$$
\begin{aligned}
0 & =\langle u+T x-f+\xi+\varrho, y-x\rangle \\
& \leq\langle u+T x-f, y-x\rangle+J^{\circ}(x, y-x)+G(y)-G(x), \quad \forall y \in X,
\end{aligned}
$$

which implies that $x$ is a solution of GVHVI.

We show that (i) $\Rightarrow$ (ii). Indeed, suppose that $x$ is a solution of GVHVI, which means that for some $u \in F(x)$,

$$
\langle u+T x-f, y-x\rangle+J^{\circ}(x, y-x)+G(y)-G(x) \geq 0, \quad \forall y \in X .
$$

From the fact that

$$
J^{\circ}(x, y-x)=\max \{\langle\xi, y-x\rangle: \xi \in \bar{\partial} J(x)\},
$$

we get that there exists a $\xi(x, y) \in \bar{\partial} J(x)$ such that

$$
\langle u+T x-f, y-x\rangle+\langle\xi(x, y), y-x\rangle+G(y)-G(x) \geq 0, \quad \forall y \in X .
$$

By virtue of Proposition 2.3, $\bar{\partial} J(x)$ is a nonempty convex and bounded subset in $X^{*}$, which implies that $\{u+T x-f+\xi: \xi \in \bar{\partial} J(x)\}$ is nonempty, convex and bounded in $X^{*}$. Since $G: X \rightarrow$ $\mathbf{R} \cup\{+\infty\}$ is a proper, convex and lower semicontinuous functional, it follows from Theorem 2.1 with $\varphi(\cdot)=G(\cdot)$ and the last inequality that there exists $\xi(x) \in \bar{\partial} J(x)$ such that

$$
\langle u+T x-f, y-x\rangle+\langle\xi(x), y-x\rangle+G(y)-G(x) \geq 0, \quad \forall y \in X .
$$

For the sake of simplicity, we denote $\xi=\xi(x)$. Then, by the last inequality we have

$$
G(y)-G(x) \geq\langle-(u+T x-f+\xi), y-x\rangle, \quad \forall y \in X
$$

which implies that $-(u+T x-f+\xi) \in \partial G(x)$. Thus, it follows from $\xi \in \bar{\partial} J(x)$ that

$$
0 \in u+T x-f+\xi+\partial G(x) \subset F(x)+T x-f+\bar{\partial} J(x)+\partial G(x)=(F+T-f+\bar{\partial} J+\partial G) x,
$$


which implies that $x$ is a solution to the inclusion problem $\operatorname{IP}(F+T-f+\bar{\partial} J+\partial G)$. This completes the proof.

Proof of Theorem 4.1. (Necessity) Assume that GVHVI is strongly (resp., weakly) $\alpha$-wellposed. Then there is a unique solution $x^{*}$ of GVHVI. By Lemma 4.1, $x^{*}$ also is the unique solution of inclusion problem $\operatorname{IP}(F+T-f+\bar{\partial} J+\partial G)$. Let $\left\{x_{n}\right\}$ be an $\alpha$-approximating sequence for $\operatorname{IP}(F+T-f+\bar{\partial} J+\partial G)$. Then there exist $w_{n} \in(F+T-f+\bar{\partial} J+\partial G) x_{n}, n \in \mathbf{N}$ and a nonnegative sequence $\left\{\epsilon_{n}\right\}$ with $\left\|w_{n}\right\|+\epsilon_{n} \rightarrow 0$ as $n \rightarrow \infty$, such that

$$
\left\langle w_{n}, y-x_{n}\right\rangle \geq-\epsilon_{n} \alpha\left(y-x_{n}\right), \quad \forall y \in X, n \in \mathbf{N} .
$$

And so there exist $u_{n} \in F\left(x_{n}\right), \xi_{n} \in \bar{\partial} J\left(x_{n}\right)$ and $\varrho_{n} \in G\left(x_{n}\right)$ such that

$$
w_{n}=u_{n}+T x_{n}-f+\xi_{n}+\varrho_{n} .
$$

From the definitions of the Clarke's generalized gradient for locally Lipschitz functional and the subgradient for convex functional, we obtain, by multiplying $y-x_{n}$ at both sides of the last equality (4.2), that

$$
\begin{aligned}
\left\langle u_{n}\right. & \left.+T x_{n}-f, y-x_{n}\right\rangle+J^{\circ}\left(x_{n}, y-x_{n}\right)+G(y)-G\left(x_{n}\right) \\
& \geq\left\langle u_{n}+T x_{n}-f, y-x_{n}\right\rangle+\left\langle\xi_{n}, y-x_{n}\right\rangle+\left\langle\varrho_{n}, y-x_{n}\right\rangle \\
& =\left\langle w_{n}, y-x_{n}\right\rangle \\
& \geq-\epsilon_{n} \alpha\left(y-x_{n}\right), \quad \forall y \in X, n \in \mathbf{N}
\end{aligned}
$$

(due to (4.1)). This immediately implies that $\left\{x_{n}\right\}$ is an $\alpha$-approximating sequence for GVHVI. Therefore, it follows from the strong (resp., weak) $\alpha$-well-posedness of GVHVI, that $\left\{x_{n}\right\}$ converges strongly (resp., weakly) to the unique solution $x^{*}$. Thus, the inclusion problem $\operatorname{IP}(F+$ $T-f+\bar{\partial} J+\partial G$ ) is strongly (resp., weakly) $\alpha$-well-posed.

(Sufficiency) Conversely, suppose that the inclusion problem $\operatorname{IP}(F+T-f+\bar{\partial} J+\partial G)$ is strongly (resp., weakly) $\alpha$-well-posed. Then $\operatorname{IP}(F+T-f+\bar{\partial} J+\partial G)$ has a unique solution $x^{*}$, which together with Lemma 4.1, implies that $x^{*}$ is the unique solution of GVHVI. Let $\left\{x_{n}\right\}$ be an $\alpha$-approximating sequence for GVHVI. Then there exist $u_{n} \in F\left(x_{n}\right), n \in \mathbf{N}$ and a nonnegative sequence $\left\{\epsilon_{n}\right\}$ with $\epsilon_{n} \rightarrow 0$ as $n \rightarrow \infty$, such that

$$
\left\langle u_{n}+T x_{n}-f, y-x_{n}\right\rangle+J^{\circ}\left(x_{n}, y-x_{n}\right)+G(y)-G\left(x_{n}\right) \geq-\epsilon_{n} \alpha\left(y-x_{n}\right), \quad \forall y \in X .
$$

From the fact that

$$
J^{\circ}\left(x_{n}, y-x_{n}\right)=\max \left\{\left\langle\xi, y-x_{n}\right\rangle: \xi \in \bar{\partial} J\left(x_{n}\right)\right\},
$$


we obtain that there exists a $\xi\left(x_{n}, y\right) \in \bar{\partial} J\left(x_{n}\right)$ such that

$$
\left\langle u_{n}+T x_{n}-f, y-x_{n}\right\rangle+\left\langle\xi\left(x_{n}, y\right), y-x_{n}\right\rangle+G(y)-G\left(x_{n}\right) \geq-\epsilon_{n} \alpha\left(y-x_{n}\right), \quad \forall y \in X .
$$

By virtue of Proposition 2.3, $\bar{\partial} J\left(x_{n}\right)$ is a nonempty, convex and bounded subset in $X^{*}$ which implies that $\left\{u_{n}+T x_{n}-f+\xi: \xi \in \bar{\partial} J\left(x_{n}\right)\right\}$ is nonempty, convex and bounded in $X^{*}$. So, it follows from (4.4) and Theorem 2.1, with $\varphi(x)=G(x)+\epsilon_{n} \alpha\left(x-x_{n}\right)$ which is proper, convex and lower semicontinuous, that there exists $\xi\left(x_{n}\right) \in \bar{\partial} J\left(x_{n}\right)$ such that

$$
\left\langle u_{n}+T x_{n}-f, y-x_{n}\right\rangle+\left\langle\xi\left(x_{n}\right), y-x_{n}\right\rangle+G(y)-G\left(x_{n}\right) \geq-\epsilon_{n} \alpha\left(y-x_{n}\right), \quad \forall y \in X .
$$

For the sake of simplicity, we denote $\xi_{n}=\xi\left(x_{n}\right)$. So, it follows from (4.5) that

$$
G\left(x_{n}\right) \leq G(y)+\left\langle u_{n}+T x_{n}-f+\xi_{n}, y-x_{n}\right\rangle+\epsilon_{n} \alpha\left(y-x_{n}\right), \quad \forall y \in X .
$$

Define the functional $\bar{G}_{n}: X \rightarrow \mathbf{R} \cup\{+\infty\}$ as follows:

$$
\bar{G}_{n}(y)=G(y)+P_{n}(y)+\epsilon_{n} Q_{n}(y),
$$

where $P_{n}(y), Q_{n}(y)$ are two functionals on $X$ defined by

$$
P_{n}(y)=\left\langle u_{n}+T x_{n}-f+\xi_{n}, y-x_{n}\right\rangle \text { and } Q_{n}(y)=\alpha\left(y-x_{n}\right) .
$$

Clearly, $\bar{G}_{n}$ is proper, convex and lower semicontinuous and $x_{n}$ is a global minimizer of $\bar{G}_{n}$ on $X$. Thus, $0 \in \partial \bar{G}_{n}\left(x_{n}\right)$. Since the functionals $P_{n}$ and $Q_{n}$ are continuous on $X, G$ is proper, convex and lower semicontinuous, it follows from Proposition 2.2 that

$$
\partial \bar{G}_{n}(y)=\partial G(y)+u_{n}+T x_{n}-f+\xi_{n}+\epsilon_{n} \partial Q_{n}(y) .
$$

This together with $0 \in \partial \bar{G}_{n}\left(x_{n}\right)$, implies that there exist $\varrho_{n} \in \partial G\left(x_{n}\right)$ and $\eta_{n} \in \partial Q_{n}\left(x_{n}\right)$ such that

$$
0=\varrho_{n}+u_{n}+T x_{n}-f+\xi_{n}+\epsilon_{n} \eta_{n} .
$$

We write $w_{n}:=-\epsilon_{n} \eta_{n}$ for all $n \in \mathbf{N}$. Then we obtain that

$$
w_{n}=u_{n}+T x_{n}-f+\xi_{n}+\varrho_{n} \in(F+T-f+\bar{\partial} J+\partial G) x_{n}, \quad \forall n \in \mathbf{N},
$$

and $-w_{n}=\epsilon_{n} \eta_{n} \in \epsilon_{n} \partial Q_{n}\left(x_{n}\right)$. So, it follows from the definition of the subgradient for convex functional that

$$
\epsilon_{n} Q_{n}(y)-\epsilon_{n} Q_{n}\left(x_{n}\right) \geq\left\langle-w_{n}, y-x_{n}\right\rangle, \quad \forall y \in X
$$

That is,

$$
\left\langle w_{n}, y-x_{n}\right\rangle \geq-\epsilon_{n} Q_{n}(y)=-\epsilon_{n} \alpha\left(y-x_{n}\right), \quad \forall y \in X .
$$


Next we claim that $\left\|w_{n}\right\| \rightarrow 0$ as $n \rightarrow \infty$, that is, for any $\varepsilon>0$ there exists an integer $N \geq 1$ such that $\left\|w_{n}\right\|<\varepsilon$ for all $n \geq N$. As a matter of fact, note that $X$ is reflexive, i.e., $X=X^{* *}$. We denote by $\mathscr{J}$ the normalized duality mapping from $X^{*}$ to its dual $X^{* *}(=X)$ defined by

$$
\mathscr{J}(v)=\left\{x \in X:\langle v, x\rangle=\|v\|^{2}=\|x\|^{2}\right\}, \quad \forall v \in X^{*} .
$$

Hence, for each $n \in \mathbf{N}$ there exists $j\left(w_{n}\right) \in \mathscr{J}\left(w_{n}\right)$ such that

$$
\left\langle w_{n}, j\left(w_{n}\right)\right\rangle=\left\|w_{n}\right\|^{2}=\left\|j\left(w_{n}\right)\right\|^{2} .
$$

Putting $y=x_{n}-j\left(w_{n}\right)$ in (4.7), we get

$$
\left\|w_{n}\right\|^{2} \leq \epsilon_{n} \alpha\left(-j\left(w_{n}\right)\right), \quad \forall n \in \mathbf{N} .
$$

If $\left\|w_{n}\right\| \nrightarrow 0$ as $n \rightarrow \infty$, then there exists $\varepsilon_{0}>0$ and for each $k \geq 1$ there exists $w_{n_{k}}$ such that

$$
\left\|w_{n_{k}}\right\| \geq \varepsilon_{0}
$$

This together with (4.8) and the property of the functional $\alpha$, leads to

$$
0<\varepsilon_{0} \leq\left\|w_{n_{k}}\right\| \leq \frac{\epsilon_{n_{k}}}{\left\|w_{n_{k}}\right\|} \alpha\left(-j\left(w_{n_{k}}\right)\right)=\alpha\left(-\epsilon_{n_{k}} \frac{j\left(w_{n_{k}}\right)}{\left\|w_{n_{k}}\right\|}\right) \rightarrow \alpha(0)=0 \quad \text { as } k \rightarrow \infty,
$$

which reaches a contradiction. This means that $\left\{x_{n}\right\}$ is an $\alpha$-approximating sequence for $\operatorname{IP}(F+T-f+\bar{\partial} J+\partial G)$. Since the inclusion problem $\operatorname{IP}(F+T-f+\bar{\partial} J+\partial G)$ is strongly (resp., weakly) $\alpha$-well-posed, we deduce that $\left\{x_{n}\right\}$ converges strongly (resp., weakly) to the unique solution $x^{*}$. Therefore, GVHVI is strongly (resp., weakly) $\alpha$-well-posed. This completes the proof.

Proof of Theorem 4.2. The proof is similar to that of Theorem 4.1 and so we omit it here.

Remark 4.1. Compared with Theorems 4.1 and 4.2 in [26], our Theorems 4.1 and 4.2 use the generalized variational-hemivariational inequality GVHVI in place of the variationalhemivariational inequality VHVI, the inclusion problem $\operatorname{IP}(F+T-f+\bar{\partial} J+\partial G)$ in place of the inclusion problem $\operatorname{IP}(A+T-f+\bar{\partial} J+\partial G)$ and the $\alpha$-well-posedness (resp., the $\alpha$-wellposedness in the generalized sense) in place of the well-posedness (resp., the well-posedness in the generalized sense). All in all, our Theorems 4.1 and 4.2 improve, extend and develop [26, Theorems 4.1 and 4.2] to a great extent.

\section{Concluding remarks}

In this paper, we introduce some concepts of well-posedness for a class of generalized variational-hemivariational inequalities with perturbations, which include, as special cases, 
the classical hemivariational inequalities, variational-hemivariational inequalities and generalized mixed variational inequalities. We establish some metric characterizations for the $\alpha$ well-posed generalized variational-hemivariational inequality, and give some conditions under which the generalized variational-hemivariational inequality is strongly $\alpha$-well-posed in the generalized sense in $\mathbf{R}^{\mathbf{n}}$. We also introduce the concept of $\alpha$-well-posedness for the inclusion problem and investigate the relations between the strong (resp., weak) $\alpha$-well-posedness of the generalized variational-hemivariational inequality and the strong (resp., weak) $\alpha$-wellposedness of the corresponding inclusion problem.

It is well known that there are many other concepts of well-posedness for optimization problems, variational inequalities and Nash equilibrium problems, such as Hadamard wellposedness [29], well-posedness by perturbations [3, 6] and Levitin-Polyak well-posedness [12, 31 , etc. It would be interesting to consider the problem of whether the concepts mentioned above can be extended to the generalized variational-hemivariational inequality.

\section{References}

[1] S. Carl, V. K. Le and D. Motreanu, Nonsmooth Variational Problems and Their Inequalities: Comparison Principles and Applications, Springer, Berlin, 2005.

[2] S. Carl and D. Motreanu, General comparison principle for quasilinear elliptic inclusions, Nonlinear Anal., 70 (2009), 1105-1112.

[3] L. C. Ceng, N. C. Wong and J. C. Yao, Well-posedness for a class of strongly mixed variational-hemivariational inequalities with perturbations, J. Appl. Math., (2012) Art. ID 712306, 21 pp.

[4] F. H. Clarke, Optimization and Nonsmooth Analysis, SIAM, Philadelphia, 1990.

[5] Y. P. Fang, N. J. Huang and J. C. Yao, Well-posedness of mixed variational inequalities, inclusion problems and fixed-point problems, J. Glob. Optim., 41 (2008), 117-133.

[6] Y. P. Fang, N. J. Huang and J. C. Yao, Well-posedness by perturbations of mixed variational inequalities in Banach spaces, European J. Oper. Res., 201 (2010), 682-692.

[7] F. Giannessi and A. Khan, Regularization of non-coercive quasi variational inequalities, Control Cybern., 29 (2000), 91-110.

[8] D. Goeleven and D. Mentagui, Well-posed hemivariational inequalities, Numer. Funct. Anal. Optim., 16 (1995), 909-921.

[9] D. Goeleven and D. Motreanu, Variational and Hemivariational Inequalities, Theory, Methods and Applications, Volume II: Unilateral Problems, Kluwer, Dordrecht, 2003.

[10] R. Hu and Y. P. Fang, Levitin-Polyak well-posedness by perturbations of inverse variational inequalities, Optim. Lett., 7 (2013), 343-359.

[11] X. X. Huang nad X. Q. Yang, Generalized Levitin-Polyak well-posedness in constrained optimization, SIAM J. Optim., 17 (2006), 243-258.

[12] X. X. Huang, X. Q. Yang and D. L. Zhu, Levitin-Polyak well-posedness of variational inequality problems with functional constraints, J. Glob. Optim., 44 (2009), 159-174.

[13] M. B. Lignola and J. Morgan, Well-posedness for optimization problems with constraints defined by variational inequalities having a unique solution, J. Glob. Optim., 16 (2000), 57-67.

[14] L. J. Lin and C. S. Chuang, Well-posedness in the generalized sense for variational inclusion and disclusion problems and well-posedness for optimization problems with constraint, Nonlinear Anal., 70 (2009), 36093617 . 
[15] Z. H. Liu and J. Z. Zou, Strong convergence results for hemivariational inequalities, Sci. China Ser. A, 49 (2006), 893-901.

[16] Z. H. Liu and D. Motreanu, A class of variational-hemivariational inequalities of elliptic type, Nonlinearity, 23 (2010), 1741-1752.

[17] R. Lucchetti and F. Patrone, A characterization of Tykhonov well-posedness for minimum problems with applications to variational inequalities, Numer. Funct. Anal. Optim., 3 (1981), 461-476.

[18] S. Migorski, A. Ochal and M. Sofonea, Nonlinear Inclusions and Hemivariational Inequalities: Models and Analysis of Contact Problems, Springer, New York, 2013.

[19] D. Motreanu and P.D. Panagiotopoulos, Minimax Theorems and Qualitative Properties of the Solutions of Hemivariational Inequalities, Kluwer, Dordrecht, 1999.

[20] Z. Naniewicz and P.D. Panagiotopoulos, Mathematical Theory of Hemivariational Inequalities and Applications, Marcel Dekker, New York, 1995.

[21] P. D. Panagiotopoulos, Nonconvex energy functions, hemivariational inequalities and substationarity principles, Acta Mech., 48 (1983), 111-130.

[22] D. Motreanu and P. D. Panagiotopoulos, Minimax Theorems and Qualitative Properties of the Solutions of Hemivariational Inequalities, Kluwer, Dordrecht, 1999.

[23] J. W. Peng and S. Y. Wu, The generalized Tykhonov well-posedness for system of vector quasi-equilibrium problems, Optim. Lett., 4 (2010), 501-512.

[24] A. N. Tykhonov, On the stability of the functional optimization problem, USSR J. Comput. Math. Phys., 6 (1966), 631-634.

[25] S. B. Nadler Jr., Multivalued contraction mappings, Pacific J. Math., 30 (1969), 475-488.

[26] Y. B. Xiao and N. J. Huang, Well-posedness for a class of variational-hemivariational inequalities with perturbations, J. Optim. Theory Appl., 151 (2011), 33-51.

[27] Y. B. Xiao, N. J. Huang and M. M. Wong, Well-posedness of hemivariational inequalities and inclusion problems, Taiwanese J. Math., 15 (2011), 1261-1276.

[28] E. Zeidler, Nonlinear Functional Analysis and Its Applications, vol. II, Springer, Berlin, 1990.

[29] J. Zeng, S. J. Li, W. Y. Zhang and X. W. Xue, Hadamard well-posedness for a set-valued optimization problem, Optim. Lett. 7 (2013), 559-573.

[30] T. Zolezzi, Extended well-posedness of optimization problems, J. Optim. Theory Appl., 91 (1996), $257-266$.

[31] X. B. Li and F. Q. Xia, Levitin-Polyak well-posedness of a generalized mixed variational inequality in Banach spaces, Nonlinear Anal. TMA, 75 (2012), 2139-2153.

[32] L. C. Ceng and J. C. Yao, Well-posedness of generalized mixed variational inequalities, inclusion problems and fixed-point problems, Nonlinear Anal. TMA, 69 (2008), 4585-4603.

[33] L. C. Ceng, N. Hadjisavvas, S. Schaible and J. C. Yao, Well-posedness for mixed quasivariational-like inequalities, J. Optim. Theory Appl., 139 (2008), 109-125.

[34] L. C. Ceng, H. Gupta and C. F. Wen, Well-posedness by perturbations of variational-hemivariational inequalities with perturbations, Filomat, 26 (2012), 881-895.

[35] K. Kuratowski, Topology, vols. 1-2, Academic, New York, NY, 1968.

[36] R. T. Rockafellar, Convex Analysis, Princeton University Press, Princeton, 1972.

Department of Mathematics, Shanghai Normal University, Shanghai 200234, China.

E-mail: zenglc@hotmail.com

Department of Industrial Management, Vanung University, Taoyuan, Taiwan.

E-mail: yylur@vnu.edu.tw

Center for Fundamental Science, and Research Center for Nonlinear Analysis and Optimization, Kaohsiung Medical University, Kaohsiung, 80708, Taiwan; Department of Medical Research, Kaohsiung Medical University Hospital, Kaohsiung 80708, Taiwan.

E-mail: cfwen@kmu.edu.tw 